\title{
Acciones de Casimiroa edulis (Rutacea) sobre la presión arterial media y frecuencia cardiaca en ratas albinas
}

\author{
Mildred Garcia González ${ }^{1,3}$. Enrique Freer Bustamante ${ }^{1,2}$ y Orlando Morales Matamoros \\ - Departamento de Fisiología, Escuela de Medicina, Universidad de Costa Rica. \\ 2 Centro de Investigación y Docencia, COOPESALUD R.L., San José, Costa Rica. \\ - Laboratorio de Ensayos Biológicos, Universidad de Costa Rica, San José, Costa Rica.
}

(Rec. 3-DX-1993. Acep. 8-II-1994)

\begin{abstract}
Aqueous extracts of Casimiroa edulis leaves injected intravenously to 20 nomal Spague-Dawley rats produced a rapid and transitory increase in blood pressure. The amplitude of the blood pressure rise was dose dependent. The increase was statistically significant (ps 0.01 ) at a concentration of $220 \mathrm{mOsm} /$, and was associated with a significant hear rate decrease (p5 0.01) probably secondary to barorreceptor stimuli. The application of 130 $\mathrm{mOsm} / \mathrm{h}$ of the extract did not produce a significant increase in blood pressure, which was followed by a rapid pressure normalization. The slight increase in cardiac rate during the pressure lift was probably due to sympathetic stimuli.
\end{abstract}

Key words: Casimiroa edulis, cardiovascular effects.

El estudio de compuestos farmacológicamente activos presentes en plantas utilizadas en medicina popular tiene una amplia tradición (Pittier 1957, Núñez 1975, (Anónimo 1977). Este tipo de estudios recientemente ha adquirido mayor importancia debido a la inminente extinción de numerosas especies tropicales.

Casimiroa edulis (Llave et Lex) es un árbol perteneciente a la familia Rutaceae (Montiel 1980), distribuido desde el Norte de México hasta Colombia y popularmente conocido en Costa Rica como "matasano". Sus frutos, de sabor agradable, han sido utilizados en medicina popular como somnífero. Son tóxicos si se ingieren en exceso, lo que refleja su nombre popular. Otras dos especies del género Casimiroa, a saber $C$. sapota y $C$. tetrameria, se han confundido a menudo con $C$. edulis debido a la similitud de su fruto.

La composición química de extractos de semillas de $C$. edulis ha sido ampliamente investigada desde la década de los 50 (Meisels y Sondheimer 1957, Sondheimer y Meisels 1958, Sondheimer et al. 1959, Mechoulan et al. 1961, Dalta et al. 1969, Panzica y Towsend 1973).
La administración endovenosa de extractos de semillas en perros, produce un efecto hipotensor, analgésico y depresor del sistema nervioso, con paro respiratorio y muerte por sobredosis (Anónimo) 1977, Lozoya et al. 1977, Lozoya y Enríquez 1981). La administración de extractos de semillas en forma oral ha demostrado también una acción hipotensora en el animal intacto (Lozoya et al. 1978). El uso de extractos de hojas no ha sido documentado como se ha hecho con las semillas.

El objetivo de este trabajo es describir los efectos en la presión arterial media (PAM) y la frecuencia cardíaca (FC) de el extracto acuoso de hojas de $C$. edulis en dos concentraciones administradas por vía endovenosa en ratas albinas normotensas anestesiadas.

\section{MATERIAL Y METODOS}

Extractos de plantas: Los extractos se prepararon con las hojas de $C$. edulis recolectadas en los jardines del Museo Nacional de Costa 
Rica durante los meses de agosto y setiembre. Para la preparación del extracto de $130 \mathrm{mOsm} / 1$ se tomaron $100 \mathrm{~g}$ de hojas frescas maceradas y se agregó $700 \mathrm{ml}$ de agua destilada. El macerado se calentó a $100^{\circ} \mathrm{C}$ durante $45 \mathrm{~min}$. El sobrenadante fue filtrado con gaza y papel filtro Watmann \#1. Para la preparación del extracto de $220 \mathrm{mOsm} / 1$, se tomó la misma cantidad del macerado de hojas frescas y se agregó $400 \mathrm{ml}$ de agua destilada, se calentó a $100^{\circ} \mathrm{C}$ durante $45 \mathrm{~min}$ y el sobrenadante se filtró con gaza y papel filtro Watmann \#1. La osmolaridad fue ajustada y medida en el extracto acuoso con un microosmómetro.

Preparación de los animales: Se utilizaron ratas adultas Sprague-Dawley de ambos sexos con un peso entre 300 y $450 \mathrm{~g}$. Los animales se mantuvieron a temperatura ambiente $\left(22^{\circ} \mathrm{C}\right.$ $26^{\circ} \mathrm{C}$ ) en sus respectivas jaulas en el bioterio de la Universidad de Costa Rica, recibiendo alimento granulado (Piensos, San José, Costa Rica) y agua potable ad libitum.

El procedimiento de anestesia se realizó por la vía i.p. con $35 \mathrm{mg} / \mathrm{kg}$ de pentobarbital sódico. Se cateterizó la vena yugular externa, y la arteria femoral, con tubos de polietileno. Para obtener el trazo electrocardiográfico se colocaron subcutáneamente electrodos de aguja en las cuatro extremidades. La frecuencia cardíaca (FC) se determinó contando las ondas $\mathrm{R}$ por minuto en la derivación $\mathrm{D}_{\text {II }}$ del electrocardiograma. Para medir los valores de la presión arterial media (PAM) el cateter en la arteria femoral se conectó al transductor de presión de un polígrafo fisiológico. Los extractos de planta fueron aplicados endovenosamente en la vena yugular externa, por medio de un catéter de polietileno (PE-50) y utilizando una bomba de infusión contínua $(1 \mathrm{ml} / \mathrm{min})$.

Se utilizaron dos grupos de animales, cada uno con 10 ratas y se les controló la PAM y la FC durante $10 \mathrm{~min}$. previamente a la administración del extracto. Este registro se utilizó como autotestigo al resultado del experimento. A cada grupo se le aplicó $0.5 \mathrm{ml}$ del extracto, el primero a una concentración de $180 \mathrm{mOsm} / 1$ y el segundo a $220 \mathrm{mOsm} / 1$. El efecto del extracto se registró en forma contínua durante $20 \mathrm{~min}$.

Los datos fueron registrados y expresados como el valor medio \pm la desviación estándar. Tratamientos y testigos fueron comparados con la distribución "T de Student", modalidad pareada.

\section{RESULTADOS}

Treinta seg después de la administración del extracto se registró un aumento rápido y transitorio de la PAM. La PAM se estabilizó en niveles basales 2 a 5 min después de la inyección. La magnitud del aumento de la presión arterial media y su duración son significativamente mayores $(p \leq 0.01)$ con el extracto de $220 \mathrm{mOsm} / 1$ que con el de $130 \mathrm{mOsm} / 1$. (Fig. 1).

Con el extracto de $220 \mathrm{mOsm} / 1$ se observó un descenso inmediato de la FC en 63 latidos/min, seguido de una recuperación a los $10 \mathrm{~min}$. El extracto de $130 \mathrm{mOsm} / 1$ no tuvo efectos significativos $(p>0.05)$ sobre la FC (Fig. 2).

Con ambos extractos, la PAM se estabilizó después del aumento inicial de la presión arterial en valores inferiores a los "valores testigo" iniciales, mostrando un efecto hipotensor tardío que se mantuvo durante $15 \mathrm{~min}$. Existe una relación proporcional entre la concentración del extracto y la magnitud del efecto hipotensor.

\section{DISCUSION}

El aumento transitorio de la presión arterial con la aplicación endovenosa de extractos de $C$. edulis, no ha sido informado hasta el momento cuando se han utilizado extractos de semillas (Sondheimer y Meisels 1958, (Anónimo) 1977, Lozoya et al. 1977, Lozoya y Enríquez 1981). Se descartó que el efecto hipertensor fuera causado por el aumento del volumen perfundido, porque con el mismo volumen de solución salina perfundido durante el registro de control previo a la administración de los extractos de la planta, no se observó aumento de la presión. Otros trabajos realizados con la misma metodología (Ramírez y Quirós 1981, Peñalba 1984, Salas 1987, Romero 1989), no mencionan aumentos de presión arterial por el aumento del volumen perfundido. La relación entre la magnitud del aumento transitorio de presión arterial y la concentración del extracto permiten sugerir que se trata de un efecto debido al extracto de $C$. edulis.

La disminución de la presión arterial registrada tras la elevación inicial mostró una relación directa con la dosis empleada. Estos resultados coinciden con los obtenidos por Lozoya y 


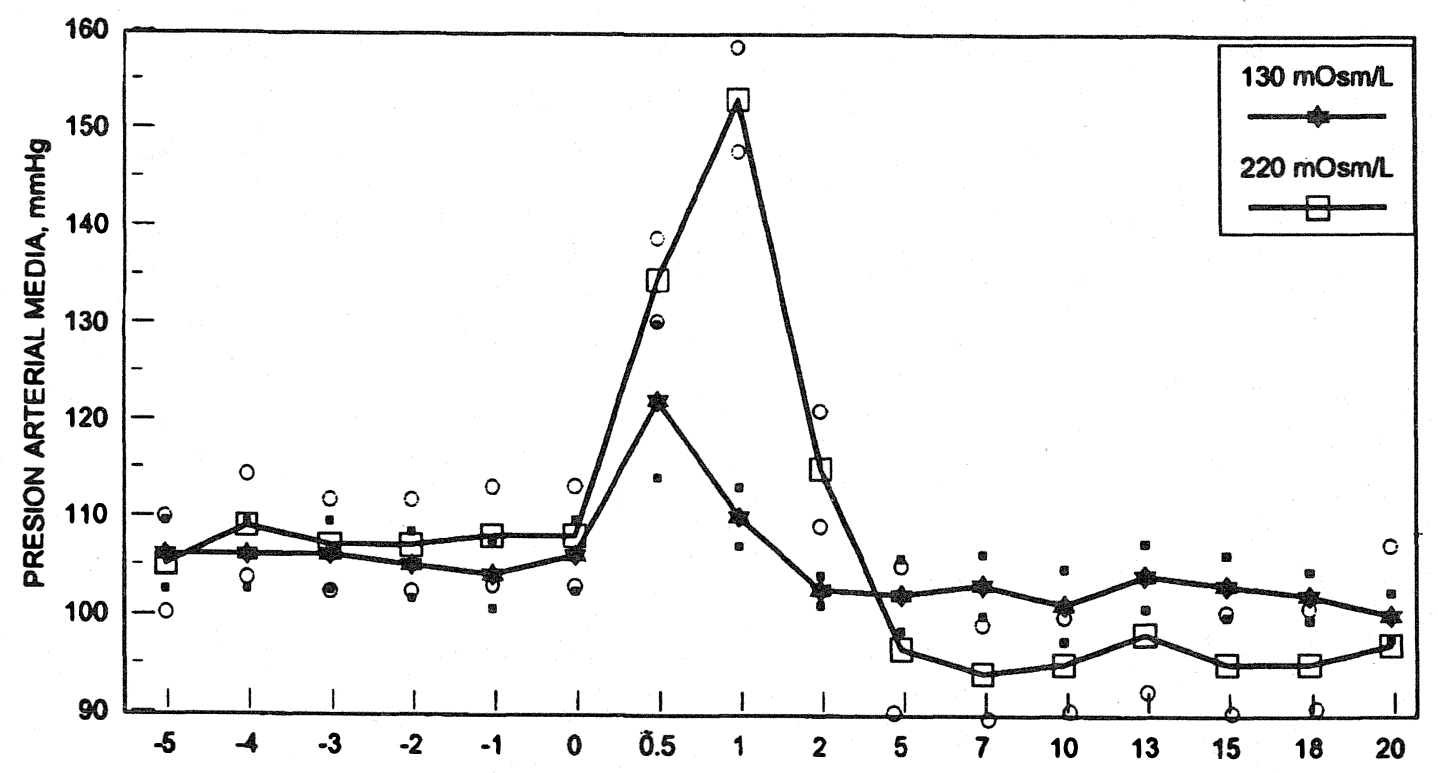

Fig. 1. Variación de la presión arterial media en ratas albinas por extractos acuosos de Casimiroa edulis.

Enríquez (1981), quienes determinaron que el grado de hipotensión es proporcional a la dosis.

Los trabajos similares hechos por el Instituto Mexicano para el Estudio de las Plantas Medicinales, (Anónimo 1977) han mencionado que el extracto alcohólico de las semillas de esta especie tiene un vigoroso efecto hipotensor con dosis tan bajas como $0.3 \mathrm{mg} / \mathrm{kg}$ de peso del extracto crudo, probablemente porque las semillas contienen mayor concentración de sustancias activas que las hojas.

Para llevar a cabo el análisis de los cambios producidos en la presión arterial por la administración de extractos de $C$. edulis, se debe tener presente conceptos de fitoquímica, ya que la síntesis de productos vegetales varía de una zona geográfica a otra, como también de acuerdo a la estación del año o a las horas del día (Thompson 1980). Estos factores podrían ser la causa de la diferencia en los resultados obtenidos por Lozoya et al.(1977) y los de la presente investigación, en cuanto a que no se ha informado un efecto hipertensor inicial.

Se debe considerar también la técnica de extracción. A diferencia de Lozoya et al. (1977), quienes extrajeron los principios activos utilizando solventes, en nuestro caso el material fue sometido a ebullición. Tradicionalmente las infusiones de semillas u hojas son administradas por vía oral, absorbiéndose estas a través de la mucosa intestinal (Lozoya et al. 1978). En otros estudios (Lozoya y Enríquez 1981) se informa que el extracto de semillas de $C$. edulis administrado por gastroclisis tenía iguales efectos que administrado en forma endovenosa, siendo los efectos en éste último caso inmediatos, mientras que en la aplicación intragástrica los efectos hipotensores se presentaron entre los 10 a 15 min.

Los efectos farmacológicos de los extractos alcohólicos y acuosos de semillas de C. edulis, administrados por vía endovenosa en gatos, perros y cobayos (Lozoya et al. 1977) produjeron efectos similares, mostrando estos últimos una acción menos intensa.

La hipotensión producida por los extractos de semillas de $C$. edulis se podria deber a un efecto periférico, afectando los vasos mesentéricos, carotídeos y musculares (Anónimo 1977). Basándose en el procedimiento de "circulación cruzada", se han obtenido evidencias sobre el tipo de hipotensión, siendo ésta debida a dilatación de los vasos sanguíneos (Lozoya y Enríquez 1981). 


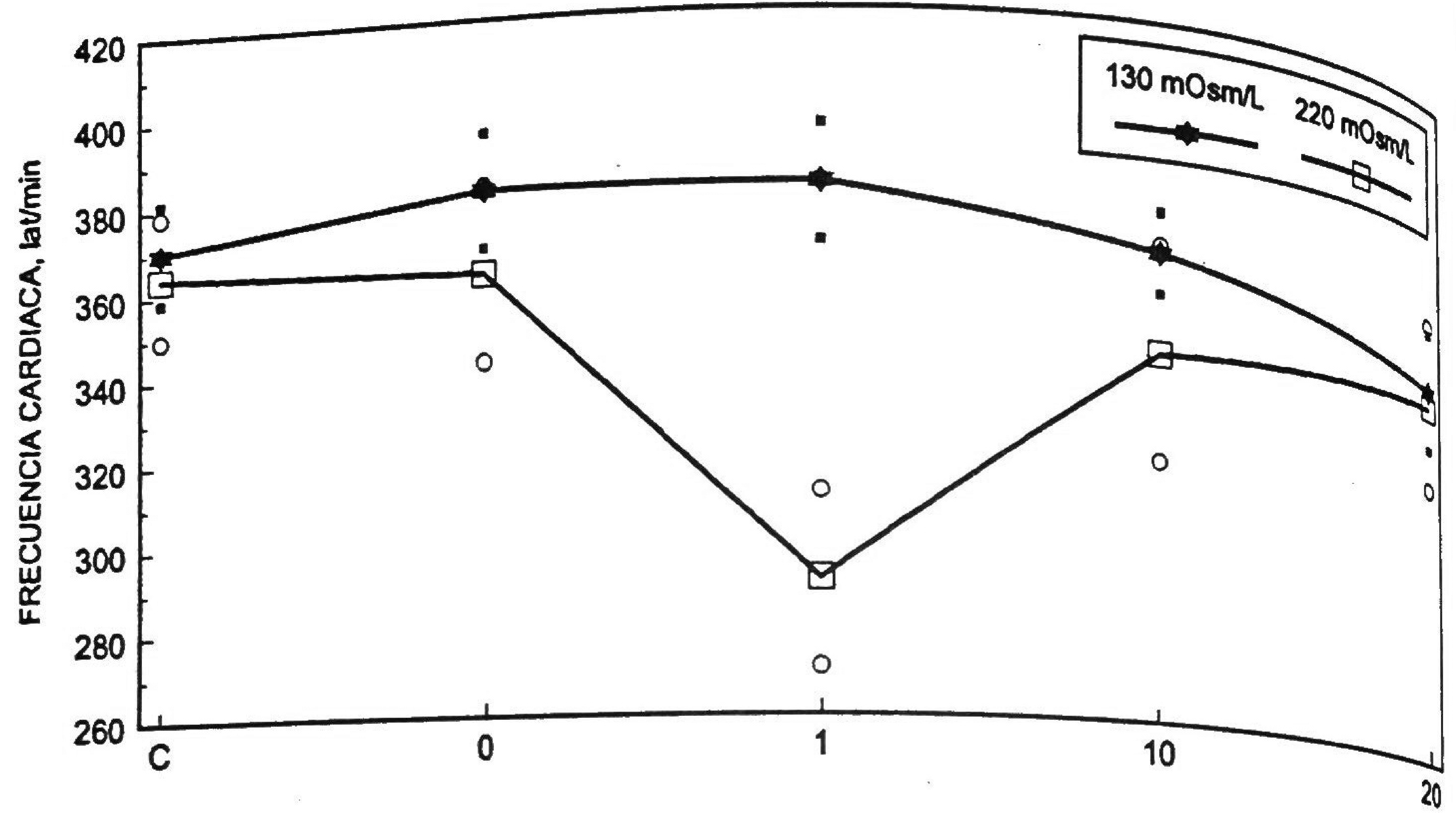

Fig. 2. Variación de la frequencia cardiaca en ratas albinas por extractos aquosos de Casimiroa edulis.

La administración del extracto acuoso a una concentración de $130 \mathrm{mOsm} / 1$, produjo un aumento no significativo de la F.C. Este aumento se produjo en el momento en que fue registrado el aumento de PAM. Esto podría explicarse por un efecto vasoconstrictor transitorio y una respuesta compensatoria vasodilatadora. Esto ya ha sido propuesto en los estudios de "circulación cruzada" (Losoya y Enríquez 1981).

La bradicardia significativa ( $\mathrm{p} \leq 0.01$ ) producida por la administración de la dosis de 220 mOsm/l, fue descrita también por Lozoya et al. (1977), utilizando dosis altas de extractos de semilla de Casimiroa. Esta respuesta bradicardizante podria explicarse por un reflejo originado en los baroreceptores, cuyas fibras aferentes viajan por los vagos, produciendo un efecto parasimpático. Con dosis de 1.5 a 3 $\mathrm{mg} / \mathrm{kg}$ se producen efectos tóxicos sobre el corazón. Como consecuencia de una descarga vagal severa que puede producir el paro cardíaco con un abatimiento brusco de la presión arterial. La muerte por intoxicación ocurre por paro cardiaco y la recuperación de la presión arterial es a veces lograda mediante la inyección de adrenalina (Anónimo 1977).

La acción de extractos acuosos y etanólicos de semillas de $C$. edulis sobre el músculo liso de la aorta, tráquea, vejiga, íleo terminal y útero; produjo efectos inmediatos y evidentes en la contractilidad, dándose un aumento tanto en la frecuencia como en la amplitud y el tono de contracción (Lozoya et al. 1977, Lozoya y En. ríquez 1981). La respuesta aórtica es una vasode una vasodilatación.

Los estudios farmacológicos con el com. puesto aislado del extracto alcohólico de las semillas de $C$. edulis concluyeron que una sola sustancia es responsable de los efectos tanto oxitócicos e hipotensores en los animales de ensayo (perro y tejido aislado de cobayo). Estudios espectroscópicos del producto aislado de la semilla, sugieren la presencia de un derivado tipo histamínico y se ha propuesto que las propiedades hipnóticas, conocidas popularmente, pueden ser la consecuencia de un efecto indirecto, resultado de una acción compensatoria debida a la hipotensión sostenida, que al producir un efecto parasimpaticomimético, propicia la aparición del sueño como respuesta fisiologica (Lozoya et al., 1977, 1978. Lozoya y Enriquez 1981).

\section{RESUMEN}

Inyectando estractos acuosos de hojas del árbol Casimiroa edulis de manera intravenosa en 20 ratas normales (tipo Spague-Dawley) se produjo un aumento rápido pero temporal en su 
presión sanguínea. La amplitud de ese aumento dependió de la dosis. El aumento fue estadísticamente significativo $(p<0.01)$ a una concentración de $220 \mathrm{~m} 0 \mathrm{sm} / 1$, y se asoció $(p<0.01)$ con una disminución del ritmo cardiaco, probablemente secundario en relación con estímulos basorreceptores. Al aplicar $130 \mathrm{~m} 0 \mathrm{sm} / 1$ del estracto no se aumentó significativamente la presión, lo cual fue seguido de una normalización rápida. El ligero aumento del ritmo cardiaco probablemente se debió a estímulos simpáticos.

\section{REFERENCIAS}

Anónima 1977. Medicina Tradicional. Casimiroa edulis. Ulave et Lex. Zapote blanca. Instituto Mexicano para el Estudio de las Plantas Medicinales. (I.M.E.P.L.A.M.), México,D.F., p. 1-3.

Dalta, S.C., U.S. Murti, U, \& I.R. Seshadni. 1969. A new synthesis of zapotin, zapotinin and relate of flavones. Ind. J. Chem. Sec. B. 7: 746-750.

Lozoya, X., G. Romero, M. Olmedo \& A. Bondani. 1977. Famacodinamia de los extractos acuoso y alcohólico de la semilla de Cesimiroa edulis. Arch. Inv. Méd. 8:145-154.

Lozoya, J., D. Rodríguez, J. Ortega, \& R Enríquez. 1978. Aislamiento de una sustancia hipotensora de la semilla de Casiminar edulis. Arch. Inv. Med. 9:565- 573.

Loeoya, X. \& R. Enríquez. 1981. El zapote blanco, investigación sobre una planta medicinal mexicana. Consejo Nacional de Ciencia y Tecnología. Ciencia Desarrollo (México) 20: 134-134.

Mechoulan, R., F. Sondheimer, A. Melera \& F.A. Kincl. 1961. The structure of zapotidine J. Am. Chem. Soc. 83:2022-2023.

Meisels, A. \& F. Sondheimer. 1957. The constituents of Casimiroa edulis. Llave a Lex. III. The structure of Casiminoin. J. Chem. Soc. 79:6328-6333.
Montiel, M. 1980. Introducción a la flora de Costa Rica. Universidad de Costa Rica, San José, Costa Rica. 21p.

Núñez, M.E. 1975. Plantas medicinales de Costa Rica y su folclore. Editorial Universidad de Costa Rica, San José, Costa Rica. 233 p.

Panzica, R. \& V. Townsend. 1973. The total synthesis: the alkaloid Casimiroedine, and Imidazaole Nucleside. J. Am. Chem. Soc. 95:8737-8740.

Peñalba, R. 1984. Efectos de extractos liofilizados de Momondica charantia sobre la glicemia y la presión arterial en preparaciones caninas agudas y crónicas. Tesis de Maestría, Universidad de Costa Rica,San José, Costa Rica.

Pittier, H.1957. Ensayo sobre plantas usuales de Costa Rica. Editorial Universidad de Costa Rica, San José, Costa Rica. 198 p.

Ramírez, M.F. \& R.J.L. Quirós. 1981. Estudio de las alteraciones fisiológicas provocadas por las fracciones de veneno de Botrops asper. Universidad de Costa Rica, San José, Costa Rica. 42 p.

Romero, M. 1989. Acción cardiovascular de extractos acuosos de hojas de Zyzygium jambos (L.)Alston. Tesis de Maestría, Universidad de Costa Rica, San José, Costa Rica.

Salas, I.G. 1987. Antihypertensive effect of Cecropia obtusifolia (Moraceae) leaf extract on rats. Rev. Biol. Trop. 35:127-130.

Sondheimer, F. \& A. Meisels. 1958. The constituents of Casimiroa edulis. Llave et Lex. IV. Identification of Edulein with 7-methoxy-1-methyl-2-phenyl-4-quindone. Org. Chem. 23:762-763.

Sondheimer, F., A. Meisels \& E. Kincl. 1959. Constituents of Casimiora edulis. Llave et Lex. V. Identity of Casimirolid and Obacunone. Org. Chem. 24:870-870.

Thompson, W. 1980. Guía práctica ilustrada de las plantas medicinales. Blume, Barcelona, España. 60 p. 